\title{
VOCABULÁRIO EXPRESSIVO DE CRIANÇAS COM DIFERENTES NÍVEIS DE GRAVIDADE DE DESVIO FONOLÓGICO
}

\author{
Expressive vocabulary of children \\ with differently severe grades of phonological deviation
}

Marcia de Lima Athayde (1), Queiti Carvalho ${ }^{(2)}$, Helena Bolli Mota ${ }^{(3)}$

\begin{abstract}
RESUMO
Objetivo: relacionar o desempenho de vocabulário expressivo com a gravidade do desvio, as faixas etárias e os valores de referência da prova de vocabulário utilizada. Métodos: a amostra foi constituída por 17 sujeitos com desvio fonológico, com idades entre três anos e cinco meses e oito anos e dois meses, sendo dividida em quatro grupos, baseado na análise do Percentual de Consoantes Corretas e classificação do grau de severidade conforme Shriberg \& Kwiatkowski (1982). Esse resultado permite classificar o desvio em: severo, moderadamente-severo, médio-moderado e médio. Foi aplicado o teste de linguagem ABFW - Vocabulário. Realizou-se análise de correlação entre idade, grau de severidade do desvio fonológico e resultados de referência do teste de vocabulário. Resultados: as crianças de menor gravidade de desvio fonológico obtiveram resultados superiores às demais, apesar da maioria destes resultados serem não significantes. Crianças menores apresentaram desempenho inferior às maiores, o que era esperado. Conclusões: é possível pensar que a gravidade do desvio fonológico pode estar influenciando o desempenho das crianças desta amostra em uma prova de vocabulário expressivo.
\end{abstract}

DESCRITORES: Testes de Linguagem; Vocabulário; Transtornos do Desenvolvimento da Linguagem

\section{INTRODUÇÃO}

A criança é exposta à linguagem desde as primeiras horas de vida. Ao longo dos seus primeiros anos, ela vai construindo seus primeiros passos na linguagem a fim de comunicar-se com o mundo a sua volta. Para isso, a criança constrói suas próprias estratégias, tentando dar um jeito na situação para poder dizer tudo o que tem vontade de exprimir. Para tanto, ela vai generalizar de forma abusiva a utilização de uma palavra cujos limites não entende muito bem ${ }^{1}$.

(1) Fonoaudióloga; Fonoaudióloga da Clínica Perffil, Santa Maria, RS; Mestranda do Programa Distúrbios da Comunicação Humana da Universidade Federal de Santa Maria.

(2) Fonoaudióloga em Gravataí, RS; Mestranda do curso de Letras - Linguística Aplicada da Pontifícia Universidade Católica do Rio Grande do Sul.

(3) Fonoaudióloga; Professora Adjunta do Departamento de Fonoaudiologia da Universidade Federal de Santa Maria, UFSM, Santa Maria, RS; Doutora em Letras.

Conflito de interesse: inexistente
Para todas as crianças o efeito do comprimento das palavars mostra-se significativo, sendo que as palavras curtas são adquiridas primeiro do que as mais extensas ${ }^{2}$. O período entre dois e quatro anos de idade corresponde ao desenvolvimento da capacidade de nomeação, através do léxico de verbos ${ }^{3}$. As crianças em processo de aquisição da linguagem, mais especificamente, de aquisição lexical, podem cometer uma série de desvios semânticos em função delas não terem ainda bem organizado o conjunto de traços de significação que diferenciam o uso de uma palavra de outra nos diferentes contextos linguísticos ${ }^{4,5}$.

O processo de aquisição e desenvolvimento fonológico ocorre de maneira gradual, até que haja o estabelecimento do sistema fonológico, de acordo com a comunidade linguística que a criança está inserida. A idade esperada para o estabelecimento deste sistema fonológico é até os 5 anos de idade, podendo estender-se dos 4 até, no máximo, os 6 anos de idade. Porém, para algumas crianças, o processamento das informações fonológicas 
acontece de maneira diferente do esperado. Estas crianças têm dificuldade na organização mental dos sons da língua, no estabelecimento do sistema fonológico alvo, bem como na adequação do input recebido. Estes casos são denominados desvios fonológicos (DF) ${ }^{6}$. Esta desordem é caracterizada por uma produção anormal dos sons e uso inadequado das regras fonológicas da língua, sendo que a causa desta ainda não está definida e sua etiologia é bastante discutida ${ }^{\text {? }}$.

A severidade e a inteligibilidade da fala no desvio fonológico apresentam graus variados ${ }^{8}$. Uma forma de classificá-los quantitativamente é a proposta que tem como enfoque as produções corretas da criança, tendo como base a análise contrastiva do sistema fonológico. Esta proposta permite que se identifique quão alterado está o sistema fonológico da criança. A partir do percentual de consoantes corretas (PCC) se determina o grau de severidade dos desvios fonológicos em severo, moderado-severo, médio-moderado e médio. Fatores como idade e dados supra-segmentais, como voz e ritmo da fala, são levados em conta para enquadrar a criança em uma das classificações de severidade, quando o valor do PCC encontra-se no limite entre duas classificações ${ }^{9}$. O desvio fonológico ocorre com grau moderado a severo em aproximadamente 2 a 3\% das crianças entre seis e sete anos, sendo que sua ocorrência é maior nas formas mais leves ${ }^{8}$.

Alguns autores verificaram em prova de vocabulário que crianças com desvio fonológico obtiveram desempenho semelhante aos seus pares da mesma faixa etária com desenvolvimento normal de linguagem. Para tanto, concluiu-se que as capacidades semânticas das crianças com desvios fonológicos permitem a verificação da extensão das alterações e pode ser considerado um fator de exclusão de distúrbios mais abrangentes de linguagem nesta população. Dessa forma, o grau de severidade do desvio fonológico pode ser um aspecto decisivo no desempenho lexical das crianças ${ }^{10}$.

Achados sugerem que o vocabulário e a memória fonológica estão relacionados desde o início do desenvolvimento da linguagem ${ }^{11}$. A memória fonológica é um componente crítico para a aprendizagem de novas palavras por estar envolvida na formação de novas formas fonológicas de longo prazo ${ }^{12}$.

Em crianças com desvio fonológico, a avaliação da memória de trabalho mostrou que tanto o executivo central como a memória fonológica estão prejudicados ${ }^{13}$. As mesmas pesquisadoras observaram relação positiva entre o desempenho em memória fonológica e o grau de severidade do desvio fonológico, mas não deste com o executivo central. Tais resultados levaram a conclusão de que o executivo central estaria mais relacionado com a aquisição do vocabulário e seria responsável pelo processamento e armazenamento de informações ${ }^{14}$.

O objetivo principal deste artigo foi analisar o desempenho de vocabulário expressivo de crianças com diferentes graus de gravidade de desvio fonológico. Além disso, buscou-se correlacionar os resultados encontrados com as diferentes idades e, também, compará-los com os valores de referência da prova de vocabulário utilizada.

\section{MÉTODOS}

Este é um estudo de pesquisa, sendo que esta foi realizada a partir de dados clínicos de crianças participantes do projeto de pesquisa "Aplicação de diferentes modelos de terapia fonológica no tratamento de crianças com desvios fonológicos" registrados no banco de dados do mesmo.

$O$ referido projeto encontra-se em andamento no Centro de Estudos de Linguagem e Fala (CELF) do Serviço de Atendimento Fonoaudiológico (SAF) da Universidade Federal de Santa Maria, UFSM. Estes dados foram coletados no período de 24 de outubro de 2000 a 11 de setembro de 2006.

Para os sujeitos serem incluídos na amostra, foram considerados os seguintes critérios de inclusão: estarem autorizadas pelos pais e/ou responsáveis para a participação na pesquisa por meio do Termo de Consentimento Livre e Esclarecido; apresentar diagnóstico de desvio fonológico de acordo com a caracterização descrita na revisão bibliográfica; não apresentar alterações evidentes nos aspectos neurológico, cognitivo, psicológico e/ou emocional; não terem sido submetidas a tratamento fonoaudiológico anterior e não apresentarem alterações na triagem fonoaudiológica quanto à linguagem (exceto no componente fonológico), audição e sistema estomatognático. Caso as crianças falhassem em um ou mais destes critérios, a mesma era excluída da amostra.

A amostra da presente pesquisa foi composta por 14 crianças do sexo masculino e 3 do sexo feminino, totalizando 17 sujeitos com idades entre 3 anos e 5 meses e 8 anos e 2 meses, sendo a média de idade 5 anos e 6 meses, os quais receberam diagnóstico de desvio fonológico evolutivo, confirmado por meio das avaliações fonoaudiológicas e complementares, citadas a seguir. Destas, três apresentaram desvio fonológico de grau severo, uma grau moderado-severo, sete apresentaram grau médio-moderado e seis grau médio.

Todos os sujeitos participantes do projeto supracitado, inicialmente passaram por uma triagem fonoaudiológica no SAF e, em seguida, foram submetidos às seguintes avaliações fonoaudiológicas: 
avaliação informal da linguagem compreensiva e expressiva, avaliação fonológica ${ }^{15}$, avaliação do vocabulário ${ }^{16}$, avaliação da articulação (protocolo elaborado no SAF/UFSM), avaliação psicomotora (protocolo elaborado no SAF/UFSM), avaliação da consciência fonológica ${ }^{17}$, avaliação simplificada do processamento auditivo ${ }^{18} \mathrm{e}$ avaliação do sistema sensório-motor-oral (protocolo elaborado no SAF/ UFSM).

Como avaliações complementares foram realizadas: avaliação otorrinolaringológica, neurológica, psicológica e audiológica.

Estas avaliações foram realizadas com o objetivo de confirmar a hipótese de desvio fonológico evolutivo.

A coleta da amostra de fala das crianças com DF foi realizada utilizando-se a Avaliação Fonológica da Criança ${ }^{15}$.

Essa avaliação é realizada através da nomeação e fala espontânea e possibilita a eliciação de todos os fones contrastivos em todas as posições que podem ocorrer em relação à estrutura da sílaba e da palavra, obtendo-se uma amostra representativa dos sons da língua.

Através da transcrição fonética e a análise contrastiva dos dados de fala, foi determinado o grau de severidade do desvio fonológico, pelo cálculo do PCC ${ }^{9}$.

O PCC foi obtido mediante a divisão do número de consoantes corretas (NCC) pelo NCC mais o número de consoantes incorretas $(\mathrm{NCl})$, multiplicados por cem $(\mathrm{PCC}=[\mathrm{NCC} /(\mathrm{NCC}+\mathrm{NCl})] \times 100)$.

Conforme os resultados do PCC, os níveis de desvio determinados pelos autores dividem-se em: desvio severo (PCC $<50 \%$ ), desvio moderadosevero (PCC $=50$ a $65 \%$ ), desvio médio-moderado $(P C C=65$ a $85 \%)$ e desvio médio $(P C C=85$ a $100 \%)$.

Foi realizada também a avaliação de vocabulário do teste ABFW ${ }^{16}$ com o objetivo de verificar a competência lexical da criança. Por meio desta prova são avaliados nove campos conceituais: vestuário, animais, alimentos, meios de transporte, móveis e utensílios, profissões, locais, formas e cores, brinquedos e instrumentos musicais. Devem ser apresentadas as figuras correspondentes e solicitar que a criança as nomeie. Essa prova analisa as designações por vocábulos usuais (DVU), as não designações (ND) e os processos de substituição (PS) utilizados pelas crianças para alcançar a nomeação correta dos vocábulos. Para cada um dos campos conceituais há um percentual de respostas que deve ser considerado como adequado a cada faixa etária.

Primeiramente foi determinado o grau de severidade do desvio fonológico, através do cálculo do
PCC. Após, foi feita análise do desempenho obtido na prova de vocabulário ${ }^{13}$ do teste $A B F W$ pelos sujeitos com diferentes graus de severidade considerando-se o número de DVU, de ND e de PS. Foi realizada análise estatística para comparar os resultados das avaliações de vocabulário entre os diferentes graus de severidade de desvio fonológico, entre as idades dos sujeitos e com os valores de referência da prova de vocabulário utilizada.

Este projeto recebeu aprovação do Comitê de Ética em Pesquisa do Centro de Ciências da Saúde da Universidade Federal de Santa Maria sob número 073/03.

$\mathrm{Na}$ análise estatística foi utilizado o teste $\mathrm{t}$ de Student, sendo que o nível de significância foi fixado em 0,05 (5\%).

\section{RESULTADOS}

Na Tabela 1 são apresentadas as médias gerais de cada grau de severidade, para DVU, ND e PS e comparadas com os valores de referência da prova utilizada. Nesta primeira análise os sujeitos foram agrupados apenas levando-se em consideração os níveis de gravidade do desvio, desconsiderando-se suas idades. Para isto, foi utilizado o teste estatístico $t$ de Student, onde os valores de "p" menores que 0,05 são considerados significantes, sendo estes destacados com asterisco na tabela.

Observou-se que as crianças do grau severo realizaram mais não designações e as crianças dos graus moderado-severo e médio-moderado realizaram mais processos de substituição, quando comparadas com as médias da normalidade, apesar destes achados serem não significantes. Já as crianças do grau médio apresentaram médias semelhantes à normalidade.

$\mathrm{Na}$ Tabela 2 são apresentados os resultados comparativos entre as médias obtidas pelos sujeitos e as médias da normalidade, para DVU, ND e PS, levando-se em consideração os níveis de gravidade do desvio e a faixa etária das crianças. Para isto, utilizou-se o teste t de Student, em que foi calculado o t tabelado para nível de significância de 5\% e grau de liberdade igual a 8 . Sendo assim, são consideradas diferenças significantes aquelas em que o valor do $t$ calculado está entre os valores do t tabelado, ou seja, entre $-2,3060$ e $+2,3060$. Os valores significantes estão destacados com asterisco na tabela.

Observou-se que crianças da faixa etária de $\mathbf{4}$ anos com desvio fonológico de grau severo apresentaram menos designações por vocábulos usuais do que a média da normalidade, porém, este resultado não foi estatisticamente significante. As mesmas crianças realizaram mais não designações do que a normalidade, sendo este um 
Tabela 1 - Comparação geral de DVU, ND e PS nos diferentes graus de severidade com os valores de normalidade

\begin{tabular}{|c|c|c|c|c|c|c|}
\hline Grau & Comparações & t calculado & p & t tabelado ${ }^{*}$ & Média & $\begin{array}{c}\text { Média } \\
\text { Normalidade }\end{array}$ \\
\hline Severo & $\begin{array}{c}\text { DVU e } \\
\text { normalidade, } \\
\text { (4 e } 5 \text { anos) }\end{array}$ & 0,09 & 0,93 & 2,06 & 53,12 & 52,50 \\
\hline Severo & $\begin{array}{c}\text { ND e } \\
\text { normalidade, } \\
\text { (4 e } 5 \text { anos) }\end{array}$ & 0,94 & 0,36 & 2,05 & 18,32 & 13,89 \\
\hline Severo & $\begin{array}{c}\text { PS e } \\
\text { normalidade; } \\
\text { (4 e } 5 \text { anos) }\end{array}$ & $-1,29$ & 0,20 & 2,04 & 27,80 & 33,61 \\
\hline Moderado-severo & $\begin{array}{c}\text { DVU e } \\
\text { normalidade, } \\
\text { (5 anos) }\end{array}$ & 1,14 & 0,27 & 2,14 & 67,84 & 60,56 \\
\hline Moderado-severo & $\begin{array}{l}\text { ND e } \\
\text { normalidade, } \\
\text { (5 anos })\end{array}$ & $-4,36$ & $0,00^{*}$ & 2,31 & 0,00 & 10,56 \\
\hline Moderado-severo & $\begin{array}{c}\text { PS e } \\
\text { normalidade, } \\
\text { (5 anos })\end{array}$ & 0,47 & 0,64 & 2,16 & 31,77 & 28,89 \\
\hline Médio-moderado & $\begin{array}{c}\text { DVU e } \\
\text { normalidade, } \\
\text { (4, } 5 \text { e } 6 \text { anos) }\end{array}$ & 0,28 & 0,78 & 2,01 & 60,32 & 58,89 \\
\hline Médio-moderado & $\begin{array}{c}\text { ND e } \\
\text { normalidade, } \\
\text { (4, } 5 \text { e } 6 \text { anos) }\end{array}$ & $-3,03$ & $0,00^{*}$ & 2,01 & 4,69 & 12,22 \\
\hline Médio-moderado & $\begin{array}{c}\text { PS e } \\
\text { normalidade, } \\
\text { (4, } 5 \text { e } 6 \text { anos) }\end{array}$ & 1,17 & 0,25 & 2,02 & 34,33 & 28,89 \\
\hline Médio & $\begin{array}{c}\text { DVU e } \\
\text { normalidade, } \\
\text { (4 e } 6 \text { anos) }\end{array}$ & 1,03 & 0,31 & 2,03 & 65,02 & 58,06 \\
\hline Médio & $\begin{array}{c}\text { ND e } \\
\text { normalidade, } \\
\text { (4 e } 6 \text { anos) }\end{array}$ & $-1,11$ & 0,27 & 2,03 & 8,74 & 13,06 \\
\hline Médio & $\begin{array}{c}\text { PS e } \\
\text { normalidade, } \\
\text { (4 e } 6 \text { anos) }\end{array}$ & $-0,52$ & 0,61 & 2,03 & 26,39 & 28,89 \\
\hline
\end{tabular}

Legenda 1: DVU - Designação por vocábulo usual; ND - Não designação; PS - Processo de substituição. Teste estatístico utilizado: t de Student, nível de significância fixado em 0,05 (5\%). O asterisco indica os valores de p significantes $(p<0,05)$.

resultado significante. Quanto aos processos de substituição, os valores encontrados se mostram dentro do esperado. Na faixa etária de $\mathbf{5}$ anos, para o mesmo nível de gravidade, as crianças apresentaram resultado satisfatório nos três aspectos analisados, sendo as médias de DVU, ND e PS próximas às médias da normalidade.

Para o grau moderado-severo, na idade de $\mathbf{5}$ anos, mesmo a criança tendo realizado mais processos de substituição do que a média da normalidade, este resultado não foi considerado significante. Já a média de DVU está dentro da normalidade. A média de ND não pôde ser utilizada na análise estatística, pois todos os valores para média eram nulos.

No grau médio-moderado, crianças da faixa etária de 4 anos realizaram mais processos de substituição do que a média da normalidade, porém este resultado não é significante. Entretanto, realizaram designações por vocábulos usuais e não designações dentro do que se esperava. 
Tabela 2 - Comparação de DVU, ND e PS com a normalidade para cada idade e grau de severidade

\begin{tabular}{lcccccc}
\hline Grau & Faixa etária & Aspectos & t calculado & t tabelado & Média & $\begin{array}{c}\text { Média da } \\
\text { normalidade }\end{array}$ \\
\hline Severo & 4 anos & DVU & $-0,89$ & $-2,3060$ & 37,59 & 44,44 \\
Severo & 4 anos & ND & $2,40^{*}$ & 2,3060 & 30,01 & 17,22 \\
Severo & 4 anos & PS & $-1,21$ & $-2,3060$ & 31,12 & 38,33 \\
Severo & 5 anos & DVU & $-1,22$ & $-2,3060$ & 68,65 & 60,56 \\
Severo & 5 anos & ND & $-1,28$ & $-2,3060$ & 6,64 & 10,56 \\
Severo & 5 anos & PS & $-1,04$ & $-2,3060$ & 24,48 & 28,89 \\
Moderado-severo & 5 anos & DVU & 1,39 & 2,3060 & 67,84 & 60,56 \\
Moderado-severo & 5 anos & ND & - & - & 0,00 & 10,56 \\
Moderado-severo & 5 anos & PS & 0,55 & 2,3060 & 31,77 & 28,89 \\
Médio-moderado & 4 anos & DVU & 0,42 & 2,3060 & 47,80 & 44,44 \\
Médio-moderado & 4 anos & ND & $-1,85$ & 2,3060 & 8,32 & 17,22 \\
Médio-moderado & 4 anos & PS & 0,64 & 2,3060 & 43,16 & 38,33 \\
Médio-moderado & 5 anos & DVU & $-0,44$ & $-2,3060$ & 57,80 & 60,56 \\
Médio-moderado & 5 anos & ND & $-9,25^{*}$ & $-2,3060$ & 1,86 & 10,56 \\
Médio-moderado & 5 anos & PS & 1,47 & 2,3060 & 39,19 & 28,89 \\
Médio-moderado & 6 anos & DVU & 1,00 & 2,3060 & 75,37 & 71,67 \\
Médio-moderado & 6 anos & ND & 1,00 & 2,3060 & 3,88 & 8,89 \\
Médio-moderado & 6 anos & PS & 0,34 & 2,3060 & 20,65 & 19,44 \\
Médio & 4 anos & DVU & 0,92 & 2,3060 & 51,20 & 44,44 \\
Médio & 4 anos & ND & $-0,55$ & $-2,3060$ & 14,26 & 17,22 \\
Médio & 4 anos & PS & $-0,69$ & 2,3060 & 34,54 & 38,33 \\
Médio & 6 anos & DVU & $2,62^{*}$ & 2,3060 & 78,84 & 71,67 \\
Médio & 6 anos & ND & $-4,87^{*}$ & $-2,3060$ & 3,23 & 8,89 \\
Médio & 6 anos & PS & $-0,48$ & $-2,3060$ & 18,25 & 19,44 \\
\hline
\end{tabular}

Legenda 2: DVU - Designação por vocábulo usual; ND - Não designação;

PS - Processo de substituição. Teste estatístico utilizado: $t$ de Student.

O asterisco indica as diferenças significantes entre as médias obtidas pelos sujeitos e as médias da normalidade.

$\mathrm{Na}$ faixa etária de $\mathbf{5}$ anos para o grau médiomoderado, as crianças realizaram menos designações por vocábulos usuais e mais processos de substituição do que a média da normalidade, porém estes resultados não são significantes. Em relação às não designações, as mesmas crianças encontram-se dentro da normalidade.

$\mathrm{Na}$ faixa etária de 6 anos, as crianças do grau médio-moderado realizaram mais processos de substituição do que a normalidade, porém, este resultado não é significante. Já os valores de DVU e ND estão dentro da normalidade.

Para as faixas etárias de $\mathbf{4}$ anos e de $\mathbf{6}$ anos, no grau médio, os valores encontrados para DVU, ND e PS encontraram-se dentro da normalidade.

$\mathrm{Na}$ Tabela 3 são apresentadas as médias de DVU, ND e PS, dentro de um mesmo grau de severidade, nas diferentes faixas etárias. Foi utilizado o teste $\mathrm{t}$ de Student, sendo considerados significantes os valores de "p" menores que 0,05, destacados com asterisco na tabela.

No grau severo, pôde-se observar que a média de DVU - 4 anos - é menor que a média DVU - 5 anos - e a média de ND - 4 anos - é maior que a média de ND - 5 anos, sendo estes dois resultados significantes. Já a média de PS - 4 anos - também é maior que a média de PS - 5 anos, porém, este achado não é significante.

Analisando-se o grau médio-moderado, observa-se que a média de DVU - 4 anos é menor que a média de DVU - 5 anos e as médias de ND e PS - 4 anos são maiores que as médias de ND e PS - 5 anos. Porém, estes não são resultados significantes. Semelhante ocorre ao se comparar as idades de 4 e 6 anos deste mesmo nível de gravidade. Porém, nesta análise as diferenças entre as médias de DVU e PS são agora significantes. Ao se comparar as idades de 5 e 6 anos deste mesmo nível de gravidade, constata-se que as médias de DVU e ND - 
Athayde ML, Carvalho Q, Mota HB

Tabela 3 - Comparação entre as idades para cada grau de severidade

\begin{tabular}{lcccccc}
\hline Grau e faixa etária & $\begin{array}{c}\text { Média } \\
\mathbf{4} \text { anos }\end{array}$ & $\begin{array}{c}\text { Média } \\
\mathbf{5} \text { anos }\end{array}$ & $\begin{array}{c}\text { Média } \\
\mathbf{6} \text { anos }\end{array}$ & $\begin{array}{c}\mathbf{t} \\
\text { calculado }\end{array}$ & $\mathbf{t}$ tabelado & $\mathbf{p}$ \\
\hline Severo DVU (4 e 5 anos) & 37,59 & 68,65 & - & $-3,06$ & $-2,12$ & $0,01^{*}$ \\
Severo ND (4 e 5 anos) & 30,01 & 6,64 & - & 3,81 & 2,12 & $0,00^{*}$ \\
Severo PS (4 e 5 anos) & 31,12 & 24,48 & - & 0,91 & 2,12 & 0,38 \\
Médio-Moderado DVU (4 e 5 anos) & 47,80 & 57,80 & - & $-0,98$ & $-2,12$ & 0,34 \\
Médio-Moderado ND (4 e 5 anos) & 8,32 & 1,86 & - & 1,32 & 2,12 & 0,22 \\
Médio-Moderado PS (4 e 5 anos) & 43,16 & 39,19 & - & 0,39 & 2,12 & 0,70 \\
Médio-Moderado DVU (4 e 6 anos) & 47,80 & - & 75,87 & $-3,11$ & $-2,12$ & $0,01^{*}$ \\
Médio-Moderado ND (4 e 6 anos) & 8,32 & - & 3,88 & 0,90 & 2,12 & 0,39 \\
Médio- Moderado PS (4 e 6 anos) & 43,16 & - & 20,65 & 2,71 & 2,12 & $0,02^{*}$ \\
Médio-Moderado DVU ( 5 e 6 anos) & - & 57,80 & 75,37 & $-2,42$ & $-2,12$ & $0,03^{*}$ \\
Médio-Moderado ND (5 e 6 anos) & - & 1,86 & 3,88 & $-1,35$ & $-2,12$ & 0,20 \\
Médio-Moderado PS (5 e 6 anos) & - & 39,19 & 20,65 & 2,36 & 2,12 & $0,04^{*}$ \\
Médio DVU (4 e 6 anos) & 51,20 & - & 78,84 & $-3,52$ & $-2,12$ & $0,01^{*}$ \\
Médio ND (4 e 6 anos) & 14,26 & - & 3,23 & 1,99 & 2,12 & 0,08 \\
Médio PS (4 e 6 anos) & 34,54 & - & 18,25 & 2,70 & 2,12 & $0,02^{*}$ \\
\hline
\end{tabular}

Legenda 3: DVU - Designação por vocábulo usual; ND - Não designação;

PS - Processo de substituição. Teste estatístico utilizado: $t$ de Student.

$O$ asterisco indica os valores de $p$ significantes $(p<0,05)$.

5 anos são menores do que as médias DVU e ND 6 anos, sendo que apenas a diferença entre as médias de DVU é significante. Ainda, nota-se que a média de PS - 5 anos é maior que a média de PS 6 anos, sendo este um achado significante.

Para o grau médio, encontrou-se que a média de DVU - 4 anos é menor que a média de DVU 5 anos, sendo este um resultado significante. Já as médias de ND e PS - 4 anos são maiores que as médias de ND e PS - 5 anos, porém, apenas a diferença entre as médias de PS são significantes.

Neste parâmetro, não foi analisado o grau moderado-severo, pois este era composto por apenas um indivíduo.

\section{DISCUSSÃO}

Apesar dos resultados não serem significantes, observou-se que as crianças do grau severo ralizaram mais não designações do que a normalidade, enquanto que os sujeitos do grau moderado-severo e médio-moderado ralizaram mais processos de substituição. Em contrapartida, as crianças do grau médio apresentaram resultados dentro do esperado para a normalidade (Tabela 1).

Por esta primeira análise pode-se pensar que as crianças do grau médio apresentam um melhor desempenho em prova de vocabulário, visto que foram as únicas a apresentarem resultados dentro do previsto pela normalidade. Sendo assim, pode-se pensar que, de certa forma, os níveis de gravidade do desvio fonológico influenciaram o desempenho das crianças desta amostra no teste de vocabulário em questão.

Este indício vai ao encontro do esperado pelas autoras deste estudo, pois era previsto que as crianças com menor gravidade de desvio fonológico apresentassem desempenho superior às demais.

Ao levar-se em consideração as idades dos sujeitos e os níveis de gravidade, observou-se que no grau severo as crianças da faixa etária de 4 anos realizam mais não designações do que a normalidade, as crianças de 5 anos do grau moderadosevero e 4, 5 e 6 anos do grau médio moderado realizaram mais processos de substituição do que o previsto pela normalidade, sendo que os sujeitos de 5 anos do grau médio-moderado apresentaram menos designações por vocábulos usuais do que o esperado para a idade. Porém, apenas o achado referente ao grau severo é significante. Em contrapartida, as crianças do grau médio de 4 e 6 anos apresentaram médias semelhantes às da normalidade nas três classes de processos de designações (Tabela 2).

Apesar de que, em sua maioria, estes resultados não são significantes, é possível pensar, mais uma vez, que o desvio fonológico pode estar influenciando o desempenho destas crianças no teste em questão. 
Uma pesquisa longitudinal semelhante vai ao encontro do presente estudo ao constatar que o desempenho em vocabulário receptivo de indivíduos com alteração de fala foi significativamente menor do que o desempenho do grupo controle na idade de 5 anos ${ }^{19}$.

Porém, estes achados diferem de outros estudos que mostraram não haver relação direta entre desenvolvimento fonológico e aquisição lexical ${ }^{10,20}$.

Ainda, este indício observado na presente pesquisa discorda do encontrado em outro estudo, em que foi constado que mesmo que o indivíduo apresente número significante de alterações fonológicas, esta alteração está apenas na representação fonológica de um item lexical, sugerindo que o acesso e a representação semântica não estão alterados ${ }^{21}$.

Comparando-se os desempenhos entre as faixas etárias de crianças de mesmo nível de gravidade de desvio fonológico, observou-se que, em geral, as crianças mais novas realizam menos designações por vocábulos usuais e mais não designações e processos de substiuição do que as crianças mais velhas, independente da gravidade do desvio (Tabela 3).

Este achado fortalece e corrobora a expectativa das autoras do presente estudo, visto que era espe- rado que as crianças de menor idade apresentassem desempenho inferior do que as maiores.

Algumas pesquisas reforçam essa relação, demonstrando que crianças de maior idade apresentam desempenho superior em prova de vocabulário, quando comparadas com aquelas de menor idade ${ }^{22,23}$.

\section{CONCLUSÃO}

Através destes resultados, é possível se pensar que a gravidade do desvio fonológico pode estar influenciando o desempenho das crianças desta amostra em prova de vocabulário, visto que os sujeitos de menor gravidade apresentaram resultados melhores do que os demais. Além disto, constatou-se que as crianças de menor idade apresentam desempenho inferior às maiores, o que era esperado.

Diante do anteriormente exposto e levando-se em consideração os resultados não significantes, sugere-se que outros estudos que envolvam a análise comparativa da relação do vocabulário expressivo com a gravidade do desvio fonológico sejam realizados de modo a confirmarem ou não estes achados.

\begin{abstract}
Purpose: to relate task performance of expressive vocabulary with the deviation severity, the age range and the reference values of the vocabulary testing used. Methods: the sample was made up of 17 subjects with phonological deviation, with ages ranging between 3 years 5 months and 8 years 2 months. They were divided into four groups, based on the PCC analysis and classification regarding the severity grade according to Shriberg and Kwiatkowski (1982). This result allows for classifying the deviation into: severe, moderately-severe, medium-moderated and medium. The Test for Young Children ABFW - Vocabulary was applied. An analysis took place for correlation among age, severity grade for the phonological deviation and reference results of the vocabulary testing. Results: children with minor phonological deviation severity obtained better results than the others, despite the majority of these results not being significant. Younger children showed lower performance than expected. Conclusions: it is possible to think that the severity of phonological deviation may influence the performance of children in this sample in the expressive vocabulary test.
\end{abstract}

KEYWORDS: Language Tests; Vocabulary; Language Development Disorders

\section{REFERÊNCIAS}

1. Aimard P. Les débuts du langage chez l'enfant. Paris: Robert Laffont; 1996.

2. Maekawa J, Storkel HL. Individual differences in the influence of phonological characteristics on expressive vocabulary development by young children. J Child Lang. 2006; 33(3):439-59.
3. Tonietto L, Parente MAMP, Duvignau K, Gaume $B$, Bosa CA. Aquisição inicial do léxico verbal e aproximações semânticas em português. Psicol Reflex Crít. 2007; 20(1):114-23.

4. Clark EV. The lexicon in acquisition. Cambridge: Cambrigde University Express; 1993.

5. Clark EV. Metalanguage directions and their uptake in language acquisition. In: Annals VIII 
International Congress for Study of Child Language. 1999 Jul 16; San Sebastian (Basque Country).

6. Vieira MG, Mota HB, Keske-Soares M. Relação entre idade, grau de severidade do desvio fonológico e consciência fonológica. Rev Soc Bras Fonoaudiol. 2004; 9(3):144-50.

7. Wertzner HF, Amaro L, Galea DES. Phonological performance measured by speech severity indices compared with correlated factors. Sao Paulo Med J. 2007; 125(6):309-14.

8. Wertzner HF. Fonologia: desenvolvimento e alterações. In: Ferreira LP, Befi-Lopes D, Limongi SC. Tratado de fonoaudiologia. São Paulo: Roca; 2004. p. 772-86.

9. Shriberg LD, Kwiatkowski J. Phonological disorders I: a diagnostic classification system. J Speech Hear Dis. 1982; 47(3):226-41.

10. Befi-Lopes DM, Gândara J. Desempenho em prova de vocabulário de crianças com diagnóstico de alteração fonológica. Rev Soc Bras Fonoaudiol. 2002; 7(1):16-23.

11. Hoff E, Core C, Bridges K. Non-word repetition assesses phonological memory and is related to vocabulary development in 20 - to 24 -month-olds. $\mathrm{J}$ Child Lang. 2008; 35(4):903-16.

12. Majerus S, Poncelet $M$, Van der Linden $M$, Weekes BS. Lexical learning in bilingual adults: the relative importance of short-term memory for serial order and phonological knowledge. Cognition. 2008; 107(2):395-419.

13. Linassi LZ, Keske-Soares M, Mota HB. Habilidades de memória de trabalho e o grau de severidade do desvio fonológico. Pró-Fono. 2005; 17(3):383-92. 14. Linassi LZ, Keske-Soares M, Mota HB. Memória de trabalho em crianças com desvio fonológico. Pró-Fono. 2004; 16(1):75-82.
15. Yavas M, Hernandorena CL, Lamprecht RR. Avaliação fonológica da criança: reeducação e terapia. Porto Alegre: Artes Médicas; 1991.

16. Befi-Lopes DM. Vocabulário. In: Andrade CRF, Befi-Lopes DM, Fernandes FDM, Wertzner HF. Teste de linguagem: nas áreas de fonologia, vocabulário, fluência e pragmática. Carapicuíba: Pró-Fono; 2000.

17. Capovilla AGS, Capovilla FC. Prova de consciência fonológica: desenvolvimento de dez habilidades da pré-escola à segunda série. Temas Desenvolv. 1998; 7(37):14-20.

18. Pereira LD. Processamento auditivo. Temas Desenvolv. 1993; 12(11):7-14.

19. Beitchman JH, Jiang H, Koyama E, Johnson CJ, Escobar M, Atkinson L, et al. Models and determinants of vocabulary growth from kindergarten to adulthood. J Child Psychol Psychiatr. 2008; 49(6):626-34.

20. Pereira LF. A influência do grau de severidade no desvio fonológico [tese]. São Paulo (SP): Universidade Federal de São Paulo; 2006.

21. Lahey M, Edwards J. Why do children with specific language impairment name pictures more slowly than their peers? J Speech Hear Res. 1996; 39(5):1081-98.

22. Basílio CS, Puccini RF, Silva EMK, Pedromônico MRM. Condições de vida e vocabulário receptivo em crianças de dois a cinco anos. Rev Saúde Pública. 2005; 39(5):725-30.

23. Mecca FDL. Investigação das funções da linguagem, da teoria da mente, do vocabulário e do desempenho escolar de alunos surdos. Rev Soc Bras Fonoaudiol. 2005; 10(4):250.

RECEBIDO EM: 27/08/2007

ACEITO EM: 11/08/2008

Endereço para correspondência:

Márcia de Lima Athayde

Av. Borges de Medeiros, 1900 ap. 07

Santa Maria - RS

CEP: 97015-090

E-mail: fga.marcia@yahoo.com.br 